\title{
Concomitant Gastric Acid Suppressants on the Survival of Patients with Non-Small-Cell Lung Cancer Treated with Epidermal Growth Factor Receptor Tyrosine Kinase Inhibitors: A Meta-Analysis
}

\author{
Jun Xia, ${ }^{1}$ Jiping Zhu, ${ }^{1}$ Lei Li, ${ }^{1}$ and Shiqin $X u \mathbb{D}^{2}$ \\ ${ }^{1}$ Department of Respiratory Medicine, Jiangsu Province Hospital of Chinese Medicine, \\ Affiliated Hospital of Nanjing University of Chinese Medicine, Nanjing 210029, China \\ ${ }^{2}$ Department of Anesthesiology, Women's Hospital of Nanjing Medical University, \\ Nanjing Maternity and Child Health Care Hospital, Nanjing 210004, China
}

Correspondence should be addressed to Shiqin Xu; sqxu_nje2@21cn.com

Received 14 October 2021; Revised 29 November 2021; Accepted 16 December 2021; Published 31 January 2022

Academic Editor: Bing Niu

Copyright ( $\odot 2022$ Jun Xia et al. This is an open access article distributed under the Creative Commons Attribution License, which permits unrestricted use, distribution, and reproduction in any medium, provided the original work is properly cited.

\begin{abstract}
Background. The influence of concomitant use of gastric acid suppressants (AS) on survival of patients with non-small-cell lung cancer (NSCLC) treated with epidermal growth factor receptor (EGFR) tyrosine kinase inhibitors (TKIs) is inconsistent according to previous studies. We performed a meta-analysis to evaluate the effect of additional AS in patients with NSCLC taking TKIs. Methods. Relevant observational studies were identified by a search of Medline, Embase, and Web of Science databases. Only studies with multivariate analyses were included. A random-effect model was used to combine the results. Results. Thirteen retrospective studies with 12259 patients were included. Pooled results showed that concomitant use of AS was associated with worse progression-free survival (PFS, adjusted hazard ratio (HR): $1.57,95 \%$ confidence interval (CI): 1.31 to $1.89, P<0.001$; $I^{2}=65 \%$ ) and overall survival (OS, adjusted HR: 1.38 , 95\% CI: 1.19 to $1.61, P<0.001 ; I^{2}=70 \%$ ) in NSCLC patients taking TKIs. Sensitivity analysis limited to studies including NSCLC with EGFR mutation showed consistent results (HR for PFS: 1.53, $P=0.003$; HR for OS: $1.43, P=0.001)$. Subgroup analyses indicated that the association between concomitant use of AS and poor survival was not significantly affected by the category of AS used (proton pump inhibitors or histamine type-2 receptor antagonists) or the country of the study (Asian or non-Asian, $P$ for subgroup analysis all $>0.05$ ). Conclusions. Concomitant use of AS in patients with NSCLC taking TKIs may be associated with poor survival outcomes.
\end{abstract}

\section{Introduction}

The epidermal growth factor receptor tyrosine kinase inhibitors (EGFR-TKIs) have become an effective treatment for patients with non-small-cell lung cancer (NSCLC) $[1,2]$. For patients with NSCLC with EGFR mutations, TKIs have been reported to confer better survival benefits than conventional cytotoxic anticancer therapies [3]. Gastric acid suppressants (ASs), including proton pump inhibitors (PPIs) and histamine type-2 receptor antagonists (H2RAs), are frequently prescribed in NSCLC patients to alleviate symptoms of gastroesophageal diseases $[4,5]$. It has been shown that approximately $30-50 \%$ of patients with lung cancer are using AS [6]. Since many TKIs are weak bases that exhibit $\mathrm{pH}$-dependent solubility [7], coadministration of AS may reduce the absorption of TKIs by increasing the intragastric PH. Early studies showed that the plasma concentrations of gefitinib and erlotinib, two commonly used EGFR-TKIs, were significantly lower in NSCLC patients with concomitant AS compared to those without AS $[8,9]$, which raised the hypothesis that coadministration of EGFR-TKIs with AS may compromise the efficacy of TKIS in patients with NSCLC [10]. However, results of previous studies evaluating the association between concomitant AS 
on survival of patients with NSCLC taking EGFR-TKIs showed inconsistent results [11-23]. Some studies suggested that concomitant use of AS was associated with poor survival in these patients $[11-13,16-18,20]$, while other studies did not show a significant association $[14,15,19,21-23]$. Therefore, we performed a meta-analysis to comprehensively summarize current evidence regarding the influence of concomitant AS on the survival of patients with NSCLC taking EGFR-TKIs.

\section{Methods}

We followed the Meta-analysis of Observational Studies in Epidemiology (MOOSE) [24] and Cochrane's Handbook [25] guidelines during the design, performing, and presenting of the meta-analysis.

2.1. Search of Electronic Databases. We identified studies by a systematic search of Medline, Embase, and Web of Science electronic databases using the following terms: (1) "proton pump inhibitor" OR "proton pump inhibitors" OR "acid suppressive therapy" OR "antisecretory therapy" OR "PPI" OR "anti-ulcer agent" OR "antacid” OR "acid suppressants" OR "histamine type-2 receptor"; (2) "lung cancer"; and (3) "survival” OR "mortality" OR "prognosis" OR "death” OR "recurrence” OR “collapse.” Only clinical studies published in English were selected. An additional manual check-up for the reference lists of relevant original and review articles was performed as a supplement. The last literature search was conducted on June 10, 2021.

2.2. Selection of Eligible Studies. Inclusion criteria were (1) observational studies published as full-length articles; (2) included adult patients (18 years or above) with a confirmed diagnosis of NSCLC treated with EGFR-TKIs; (3) patients with concomitant use of AS, including PPIs and H2RAs, who considered as exposure; (4) compared progression-free survival (PFS) or overall survival (OS) between patients with and without concomitant use of AS; and (5) reported relative risk for the association between concomitant use of AS and survival outcomes in multivariate analysis including possible confounding factors. The definition of concomitant use of AS was consistent with the criteria adopted in the original articles. Reviews, noncohort studies, studies including nonNSCLC patients, studies with patients not using EGFR-TKIs, or studies that did not report PFS or OS were excluded.

\subsection{Extraction of Data and Evaluation of Study Quality.} Two of the authors independently conducted electronic database search, extraction of study data, and assessment of study quality according to the inclusion criteria described above. If there were discrepancies, they were resolved by discussion with the corresponding author. The extracted data included the following: (1) name of the first author, year of the publication, study design, and country of the study; (2) population characteristics, including diagnosis, total number, mean age, and sex of the patients; (3) TKI used; (4) definition of concomitant AS use and number of AS users in each study; and (5) outcomes reported and variables adjusted in the multivariate model analyzing the association between concomitant AS use and survival outcomes. The Newcastle-Ottawa Scale (NOS) [26] was used for study quality assessment, which included three domains such as defining of study groups, between-group comparability, and validation of the outcome. This scale totally scored from 1 to 9 stars, with 9 stars indicating the highest study quality level.

2.4. Statistical Methods. The primary objective of the study was to determine the association between concomitant AS use and survival outcomes in NSCLC patients taking TKIs. Hazard ratios (HRs) and 95\% confidence intervals (CIs) were selected as the general outcome variable for the associations. Data of HRs and standard errors (SEs) were calculated from $95 \%$ CIs or $P$ values, and an additional logarithmical transformation was performed to stabilize variance and normalize to the distribution [25]. Cochrane's $Q$ test was used to evaluate the heterogeneity, and the $I^{2}$ statistic was also estimated [27]. Heterogeneity was deemed to be significant if $I^{2}>50 \%$. We used a random-effect model for data synthesis because this model has incorporated the potential between-study heterogeneity and could provide a more generalized result [25]. Sensitivity analyses were performed by omitting one individual study at a time to examine the robustness of the finding [28]. Moreover, sensitivity analysis limited to studies including patients with EGFR mutation was also performed. Influences of study characteristics on the association between concomitant AS and the survival outcomes were tested with predefined subgroup analyses, including categorizes of AS (PPIs or H2RAs), country of the study (Asian or non-Asian), and TKIs used. These variables were chosen for subgroup analyses because previous studies have suggested that differences in AS used, ethnicity of the patients, and categories of TKIs may influence the survival of survival in cancer patients receiving TKIs [29-31]. The funnel plots were constructed, and a visual inspection of the symmetry was conducted to reflect the publication bias. Begg's test and Egger's regression asymmetry test were further performed for the evaluation of potential publication bias [32, 33]. We used the RevMan (Version 5.1; Cochrane Collaboration, Oxford, UK) software for the statistical analyses.

\section{Results}

3.1. Results of Database Search. The database search process is summarized in Figure 1. Briefly, 1212 articles were found in the initial literature search of the Medline, Embase, and Web of Science databases; after excluding the duplications, 1022 studies remained. An additional 984 were excluded through screening of the titles and abstracts mainly because of the irrelevance to the metaanalysis. The remaining 38 studies underwent a full-text review. Of the 38 studies, 25 were further excluded for the reasons listed in Figure 1. Finally, thirteen cohort studies [11-23] were included. 


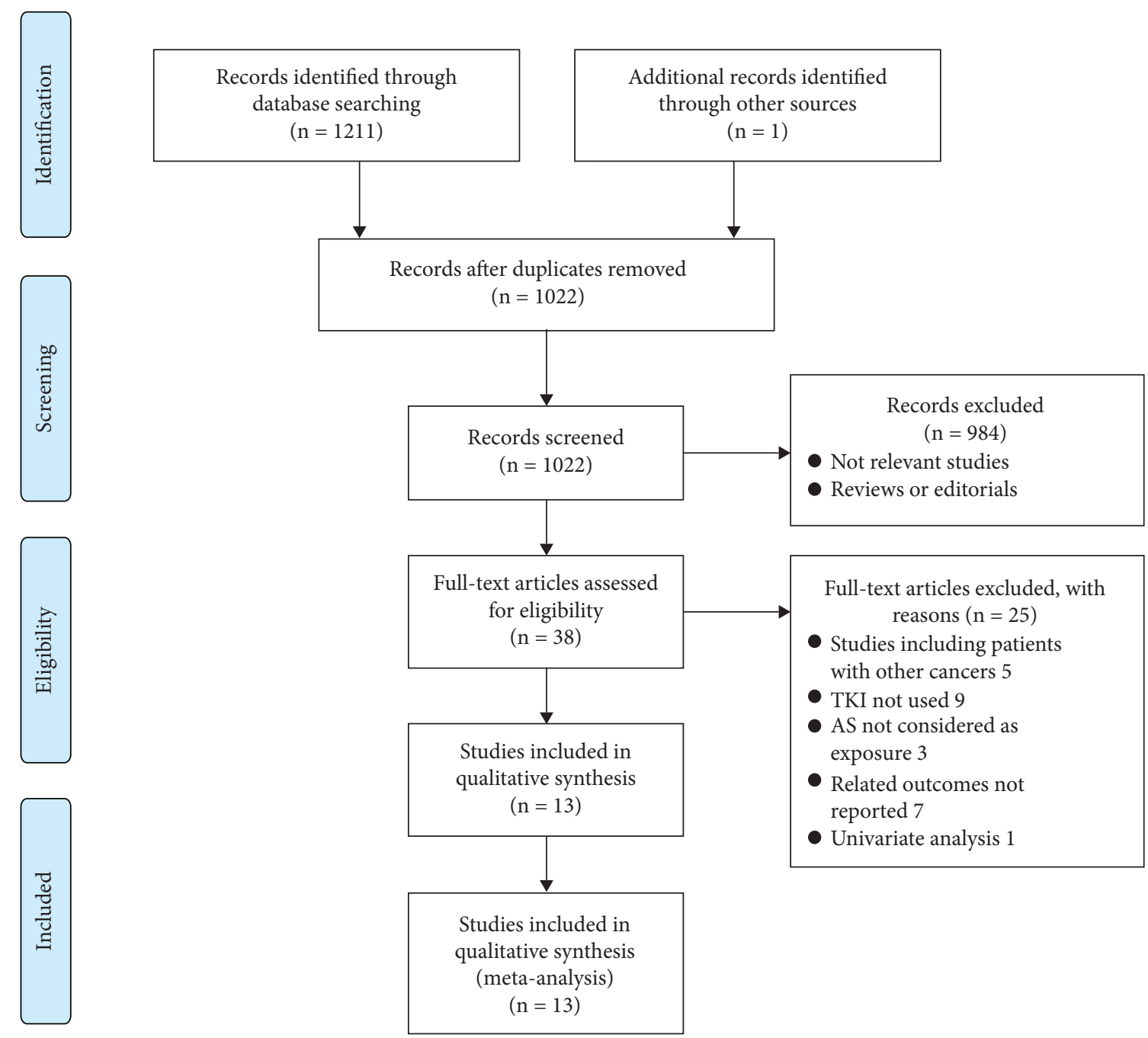

FIGURE 1: Flowchart of the database search and study identification.

3.2. Characteristics of the Included Studies. As shown in Table 1, thirteen cohort studies [11-23] including 12259 patients with NSCLC that were treated with TKIs were included. Since one study reported two cohorts of patients taking dacomitinib or gefitinib, respectively [22], these two datasets were included independently in the meta-analysis. These studies were published between 2013 and 2021 and performed in Canada [11, 12], Spain [16], the United States $[18,22]$, Japan $[15,23]$, Singapore [14], and China $[13,17,19-21]$, respectively. All of the studies were retrospective cohort studies and included patients with advanced NSCLC treated with TKIs. The mean ages of the patients varied between 61 and 76 years, and the proportions of males varied between 28 and $65 \%$. In most of the included studies, the first-generation EGFR-TKIs such as erlotinib and gefitinib were used except for two studies, in which some of the patients also used the second-generation EGFR-TKIs such as afatinib and dacomitinib [21, 22]. Concomitant use of AS was validated by medical or prescription records in most of the studies except for one study, which was self-reported [11]. Concomitant use of PPIs or H2RAs was defined as exposure in most of the included studies except for four studies, which observed the concomitant use of PPIs $[17,18,22]$ or H2RAs [23] only. Variables including age, sex, performance status, clinical stage, smoking history, comorbidities, and metastatic status were adjusted to a varying degree among the included studies. The NOS of the included studies was 7 to 9 stars, suggesting the generally good quality of the included studies (Table 2).

\subsection{Association between Concomitant AS and PFS in NSCLC} Patients Taking TKIs. Ten studies reported the association between concomitant AS and PFS in NSCLC patients taking TKIs [11-16, 19, 20, 22, 23]. Pooled results with a randomeffect model showed that concomitant AS was independently associated with a worse PFS in NSCLC patients taking TKIs (adjusted HR: 1.57, 95\% CI: 1.31 to $1.89, P<0.001$; $I^{2}=65 \%$; Figure $\left.2(\mathrm{a})\right)$. Sensitivity analyses by excluding one study at a time showed consistent results (HR: 1.49 to $1.64, P$ all <0.05). Further sensitivity analyses limited to studies including patients with EGFR mutation also showed similar results (HR: $1.53,95 \% \mathrm{CI}: 1.15$ to $2.04, P=0.003 ; I^{2}=69 \%$; Figure 2(b)). Subgroup analyses indicated that the 


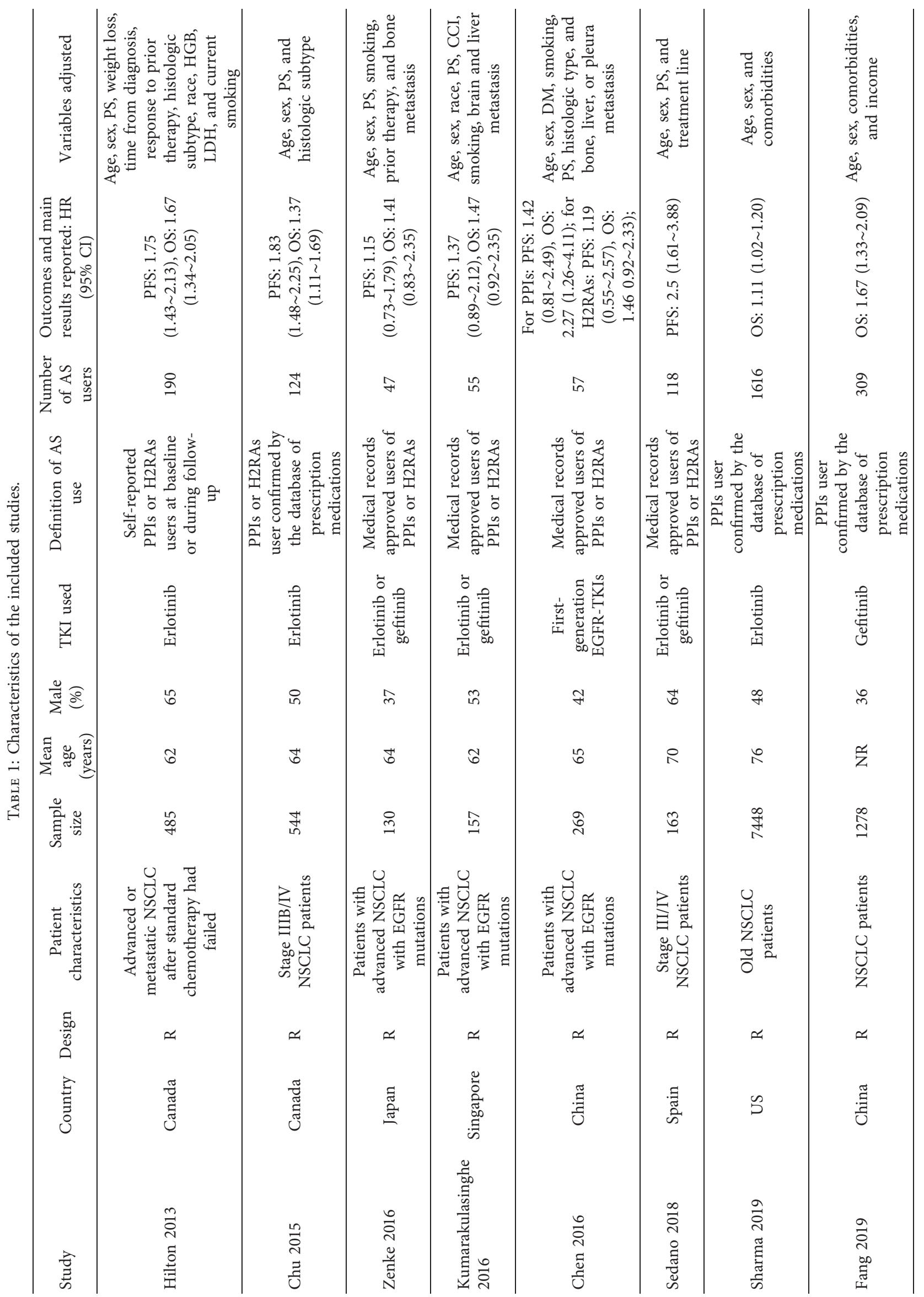




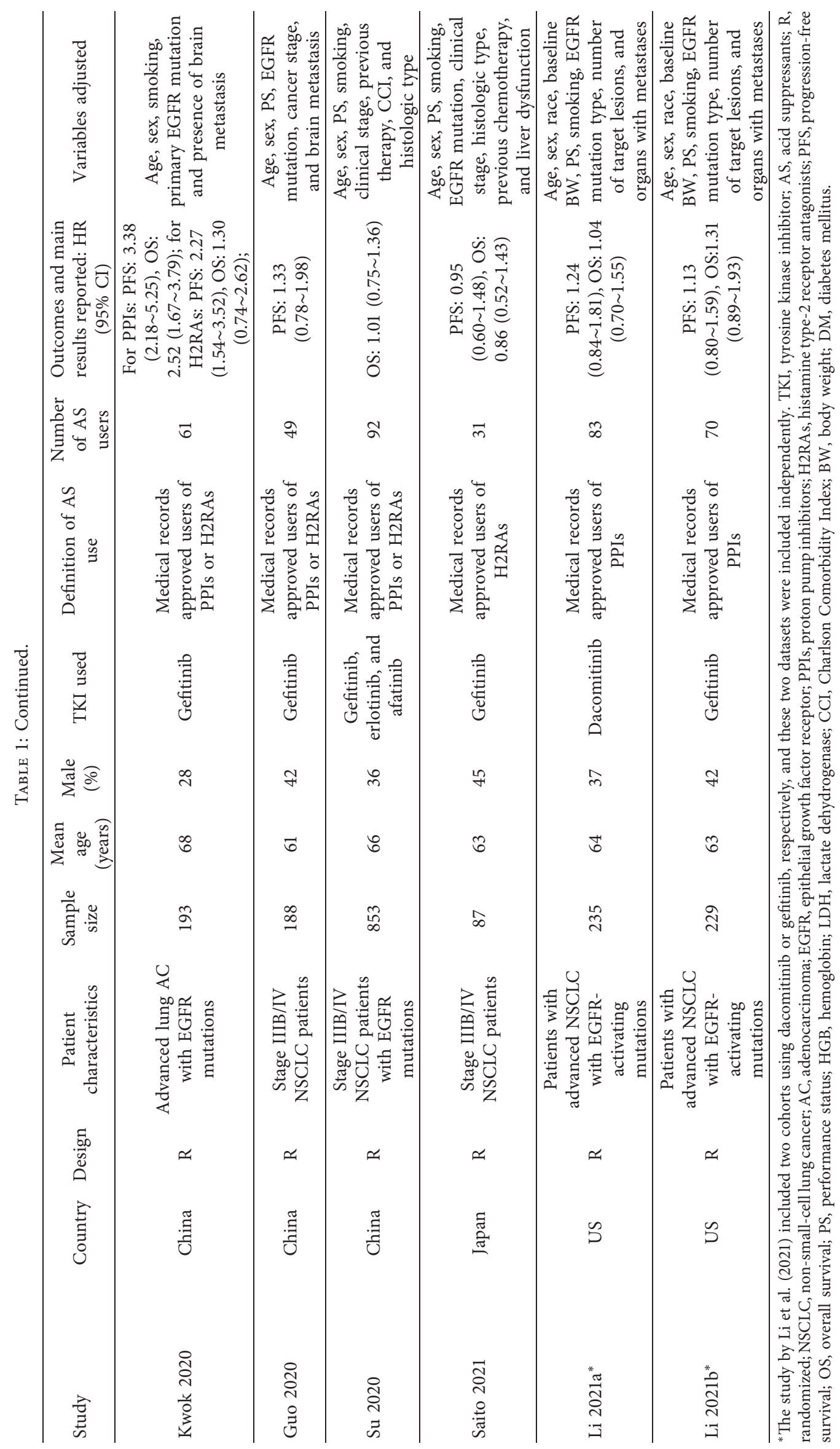




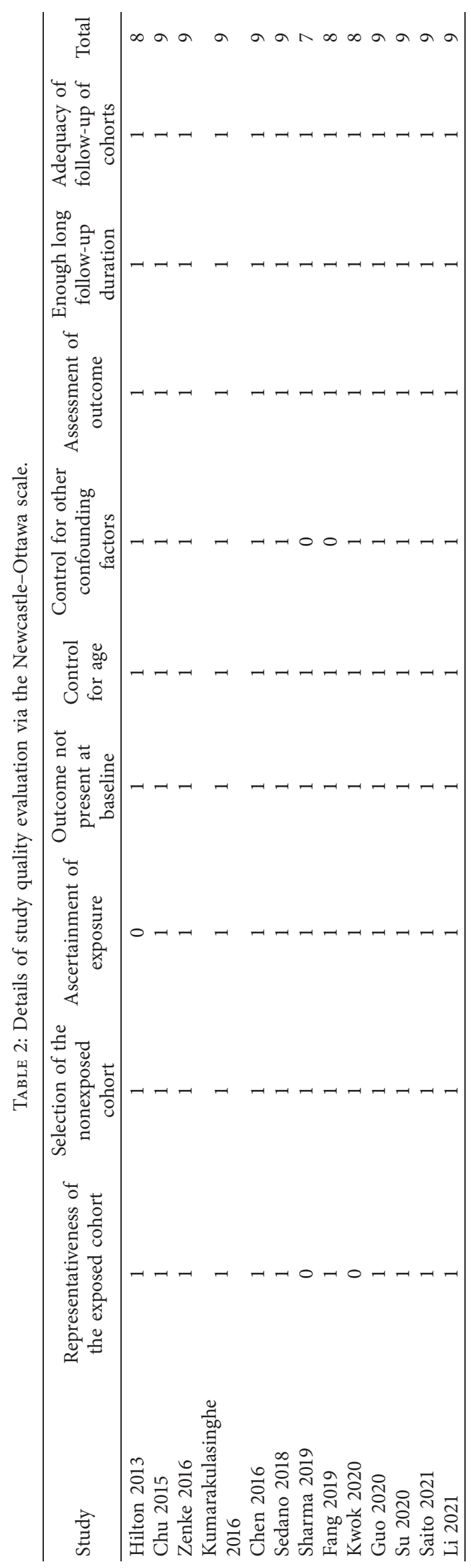


association between concomitant use of AS and worse PFS was not significantly affected by category of AS used, country of the study, or category of TKIs (Figures 2(c), 3(a), and 3(b), $P$ for subgroup difference all $>0.05)$.

\subsection{Association between Concomitant AS and OS in NSCLC} Patients Taking TKIs. Eleven studies reported the association between concomitant AS and OS in NSCLC patients taking TKIs [11-15, 17, 18, 20-23]. Results of meta-analysis showed that concomitant AS was independently associated with a worse OS (adjusted HR: 1.38, 95\% CI: 1.19 to 1.61, $P<0.001 ; I^{2}=70 \%$; Figure 4(a)), which were consistent in sensitivity analyses by omitting one study at a time (HR: 1.33 to $1.43, P$ all $<0.05$ ) and limiting to studies including patients with EGFR mutation (HR: $1.43,95 \% \mathrm{CI}: 1.15$ to 1.78 , $P=0.001 ; I^{2}=54 \%$; Figure 4(b)). Subgroup analyses also did not show a significant difference regarding the association between concomitant use of AS and OS according to the category of AS used, country of the study, or category of TKIs (Figures 4(c), 5(a), and 5(b), $P$ for subgroup difference all $>0.05)$.

3.5. Publication Bias. Figures 6(a) and 6(b) show the funnel plots regarding the meta-analyses of the associations between concomitant AS with PFS and OS in NSCLC patients taking TKIs. The visual inspection found symmetry of the plots, which suggested a low risk of publication bias. Results of Begg's tests ( $P=0.34$ and 0.77 , respectively) and Egger's regression tests $(P=0.29$ and 0.47 , respectively) also suggested the low risk of publication bias.

\section{Discussion}

In this meta-analysis, by pooling the results of available studies, we found that concomitant use of AS in NSCLC patients taking EGFR-TKIs was associated with worse PFS and OS as compared to those without AS. Further sensitivity analysis by excluding one dataset at a time and limiting to studies including patients with EGFR mutation only showed consistent results. Moreover, subgroup analysis did not show a significant different association in studies with PPIs or H2RAs, in Asian or non-Asian studies, or in studies with different TKIs. Taken together, these results suggested that concomitant use of AS may be independently associated with poor survival in NSCLC patients taking EGFR-TKIs. The combined use of AS and TKIs in patients with NSCLC should be cautious.

Several methodologic strengths of the meta-analysis should be noticed before the interpretation of the results. Firstly, an extensive search strategy was used to identify upto-date studies relevant to the aim of the meta-analysis. This expanded search strategy was applied to avoid the missing of potentially relevant studies. In addition, only studies with multivariate analyses were included, aiming to provide an independent relationship between concomitant use of AS and poor survival of NSCLC patients taking TKIs. Finally, multiple predefined sensitivity and subgroup analyses were performed to evaluate the stability of the findings. Results of sensitivity analyses indicated that the possible independent relationship between concomitant use of AS and poor survival of NSCLC patients taking TKIs was not primarily driven by either of the included studies and remained significant in patients with EGFR mutations. Results of subgroup analysis showed that the above association was not significantly affected by categories of AS, location of the study, or TKIs used. As mentioned previously, the pharmacological basis for the finding is the potential drug interaction between AS and TKIs, which causes the reduced absorptions and plasma concentrations of TKIs in patients with NSCLC and compromised anticancer efficacies [34]. Considering the solid efficacy of EGFR-TKIs in NSCLC patients with EGFR mutations and the high prevalence of AS prescription in these patients, indications of AS should be strictly followed to reduce the unnecessary combined use of TKIs and AS in these patients.

We performed multiple subgroup analyses to influence whether the difference in categorizes of AS (PPIs or H2RAs), country of the study (Asian or non-Asian), and TKIs used may affect the influence of AS on survival in NSCLC patients treated with TKIs. Although subgroup analysis was usually used to analyze the source of heterogeneity, subgroup analyses could also be performed to investigate whether the outcomes are different according to the predefined subgroup variables (test for subgroup difference). Accordingly, for subgroups with $I^{2}$ remains significant $(>50 \%)$, it may indicate that differences in predefined subgroup analyses, such as the category of AS used, country of the study, or category of TKIs, were not the major source of heterogeneity. Although our subgroup analysis did not show that category of AS had significant influences on the association between AS and poor survival in NSCLC patients taking TKIs, the associations with poor PFS and OS were significant in the subgroup of studies with PPIs but nonsignificant in the subgroup of studies with H2RAs. Although validation in large-scale prospective cohort studies is needed, these findings might suggest a less influence of H2RAs than that of PPIs on the anticancer efficacy of TKIs. This may be explained by the fact that, compared to PPIs, H2RAs generally have a shorter duration of acid-suppressive effects and achieve a lower intragastric $\mathrm{PH}$ [35], which may influence less on the absorption of TKIs. Therefore, the use of HR2As may be considered over PPIs in NSCLC patients who need a combined treatment. Besides, results of subgroup analysis according to the type of TKIs showed that the association between concomitant AS and poor survival of NSCLC patients was significant in patients taking the first-generation TKIs including gefitinib and erlotinib but not significant in patients taking dacomitinib, the second-generation TKIs. These results should be interpreted with caution since only one dataset is available for dacomitinib, and the betweensubgroup difference was not significant $(P=0.21$ for PFS and 0.38 for OS). However, the use of alternative TKIs that may be affected less by AS could be a resolution for NSCLC patients who have to use a combined treatment of AS and TKIs. For example, it has been shown that afatinib is highly soluble throughout the physiologic $\mathrm{pH}$ range of 1-7 and may therefore have fewer interactions with AS [36]. In addition, 


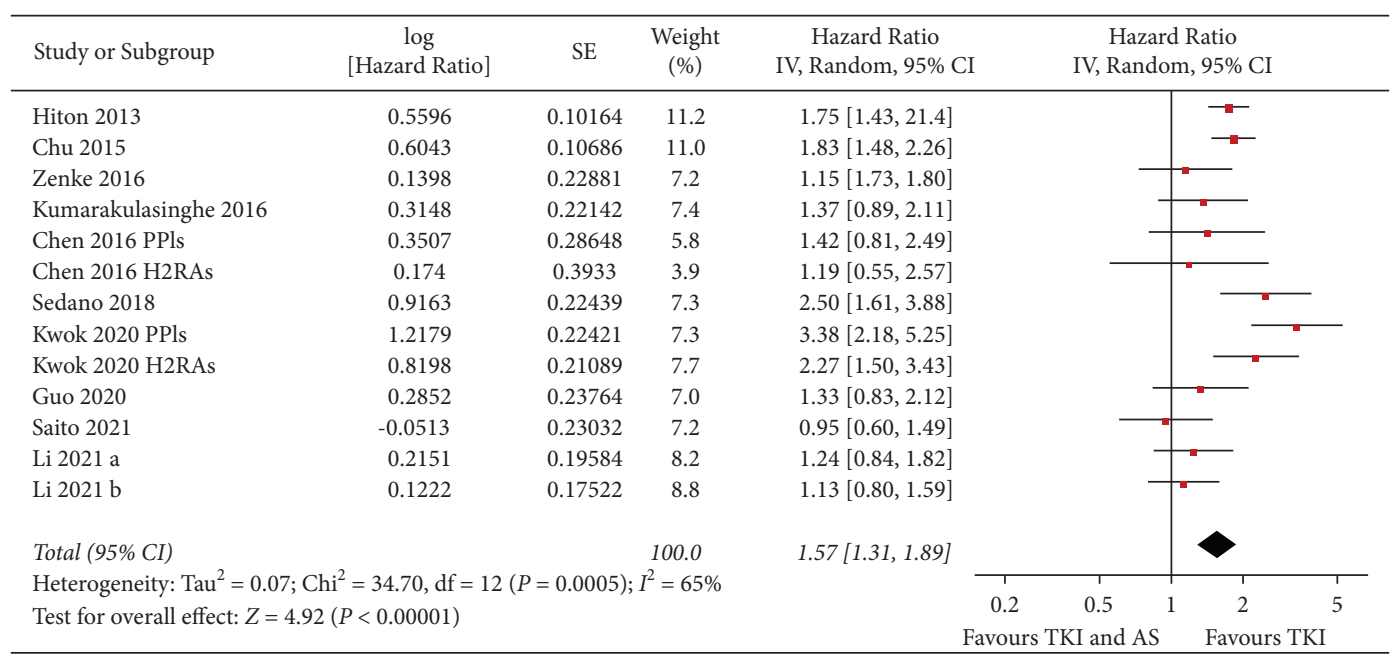

(a)

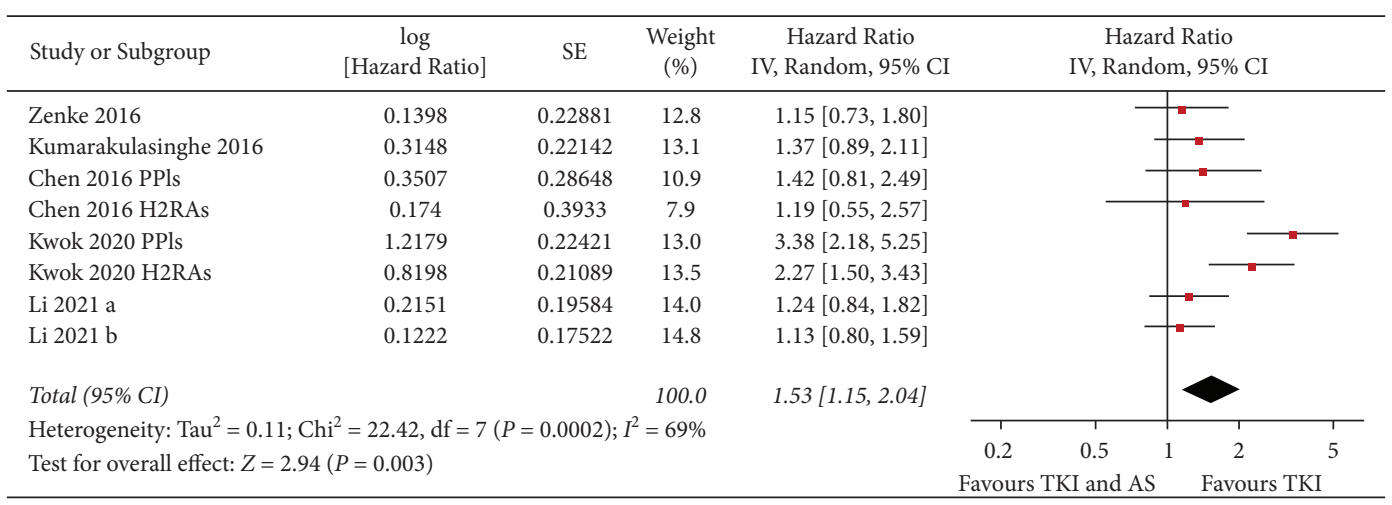

(b)

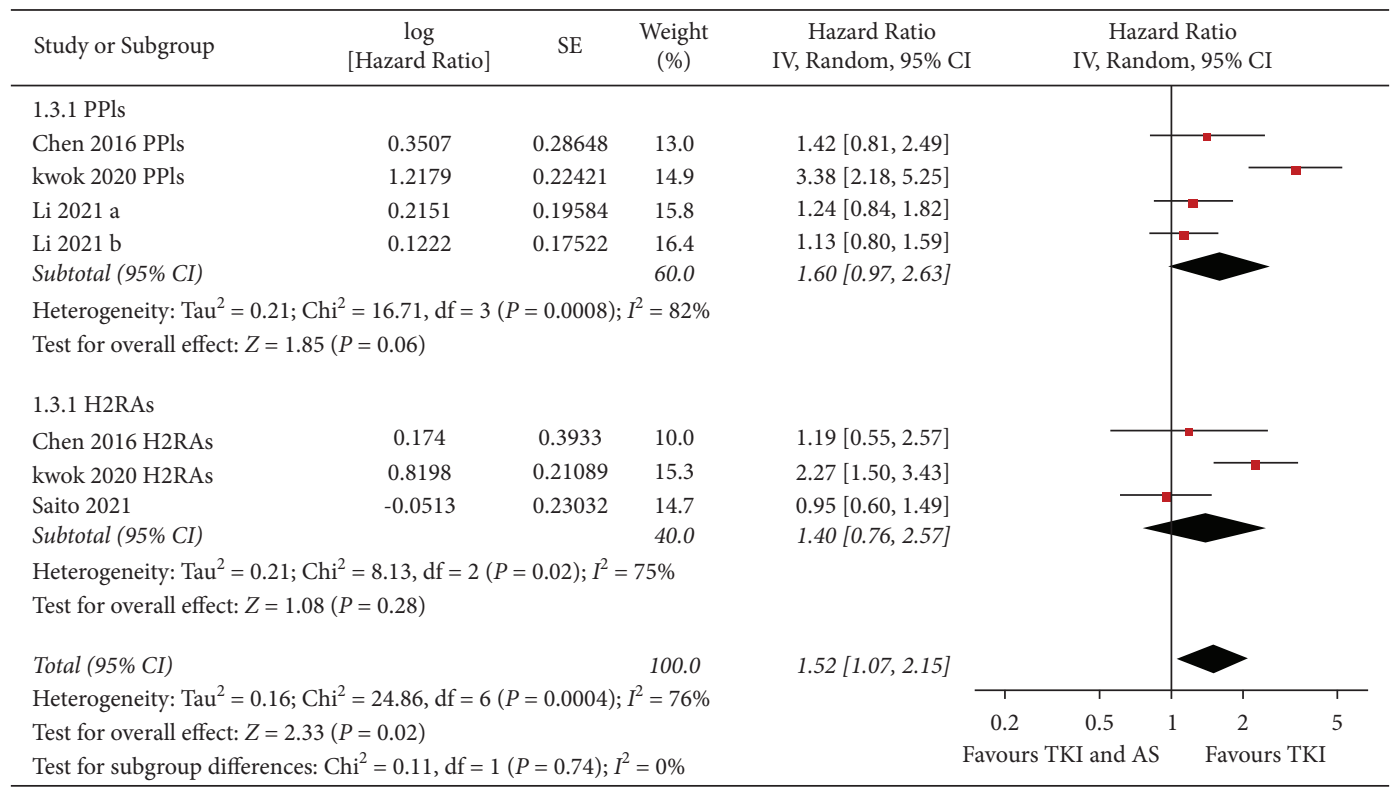

(c)

FIGURE 2: Forest plots for the meta-analysis of the association between concomitant AS use and PFS in NSCLC patients taking TKIs. (a) Forest plots for the overall meta-analysis, (b) forest plots for the sensitivity analysis in patients with EGFR mutation, and (c) forest plots for the subgroup analysis according to AS used. 


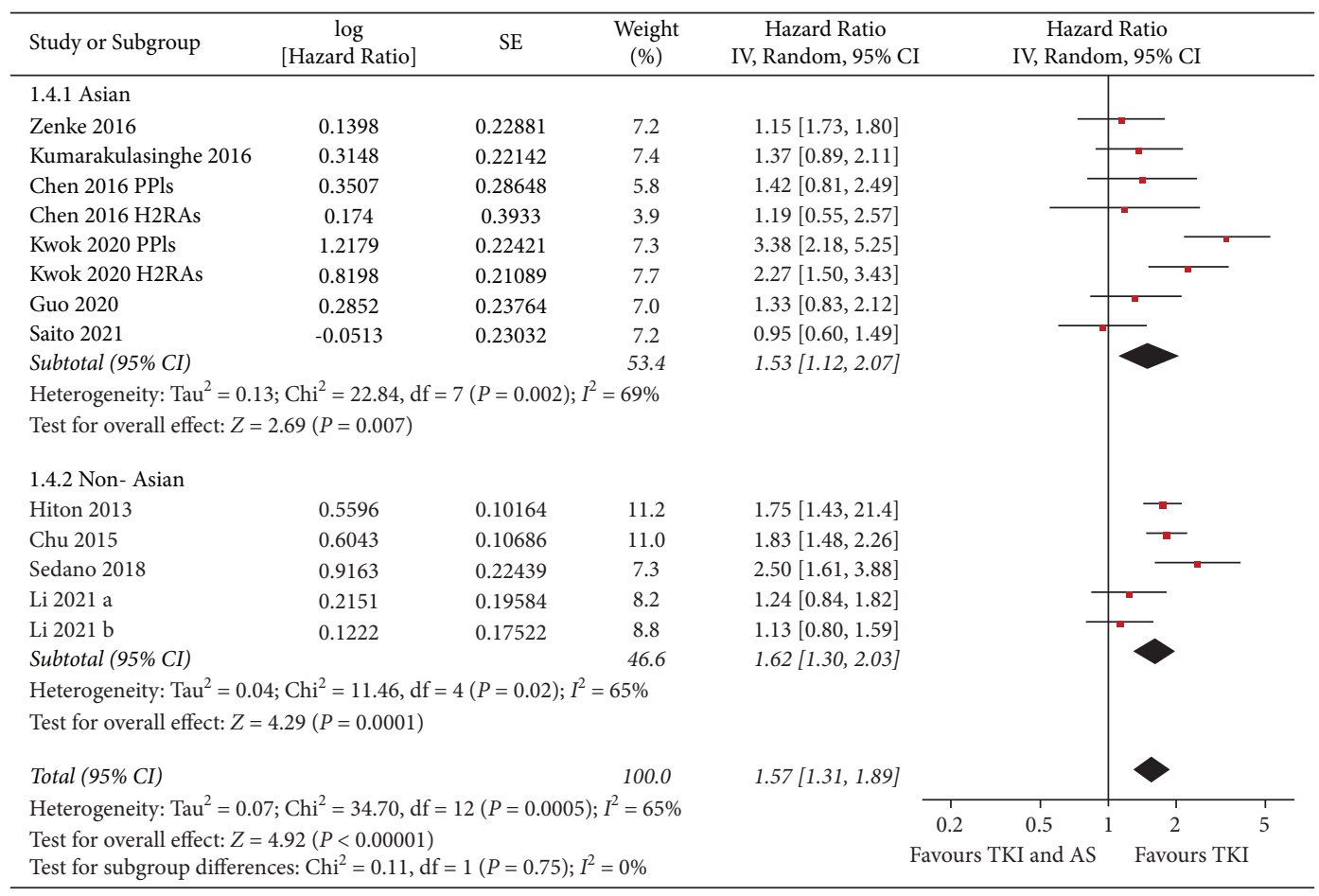

(a)

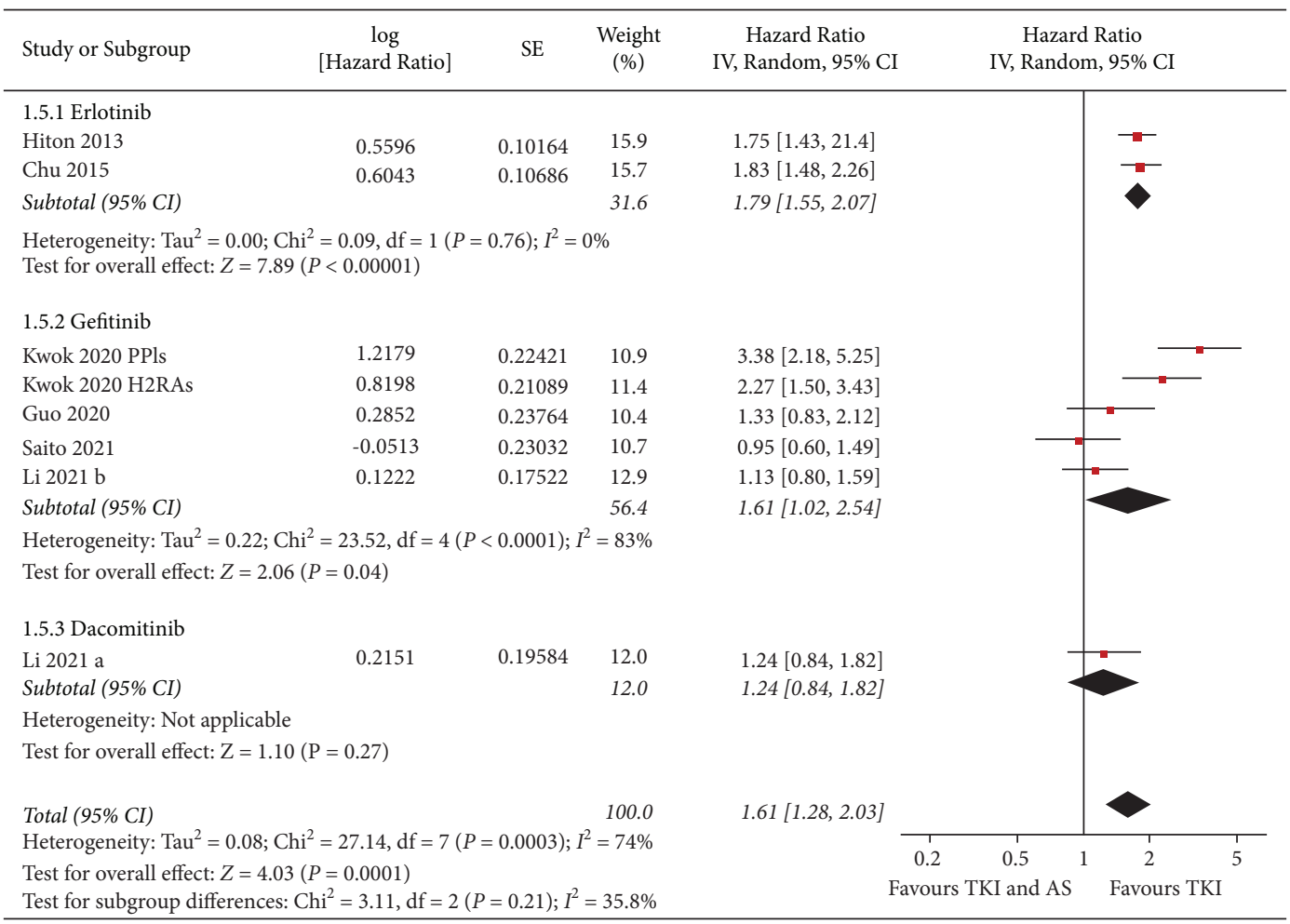

(b)

FIGURE 3: Forest plots for the subgroup analyses of the association between concomitant AS use and PFS in NSCLC patients taking TKIs. (a) Subgroup analysis according to the country of the study and (b) subgroup analysis according to TKIs used. 


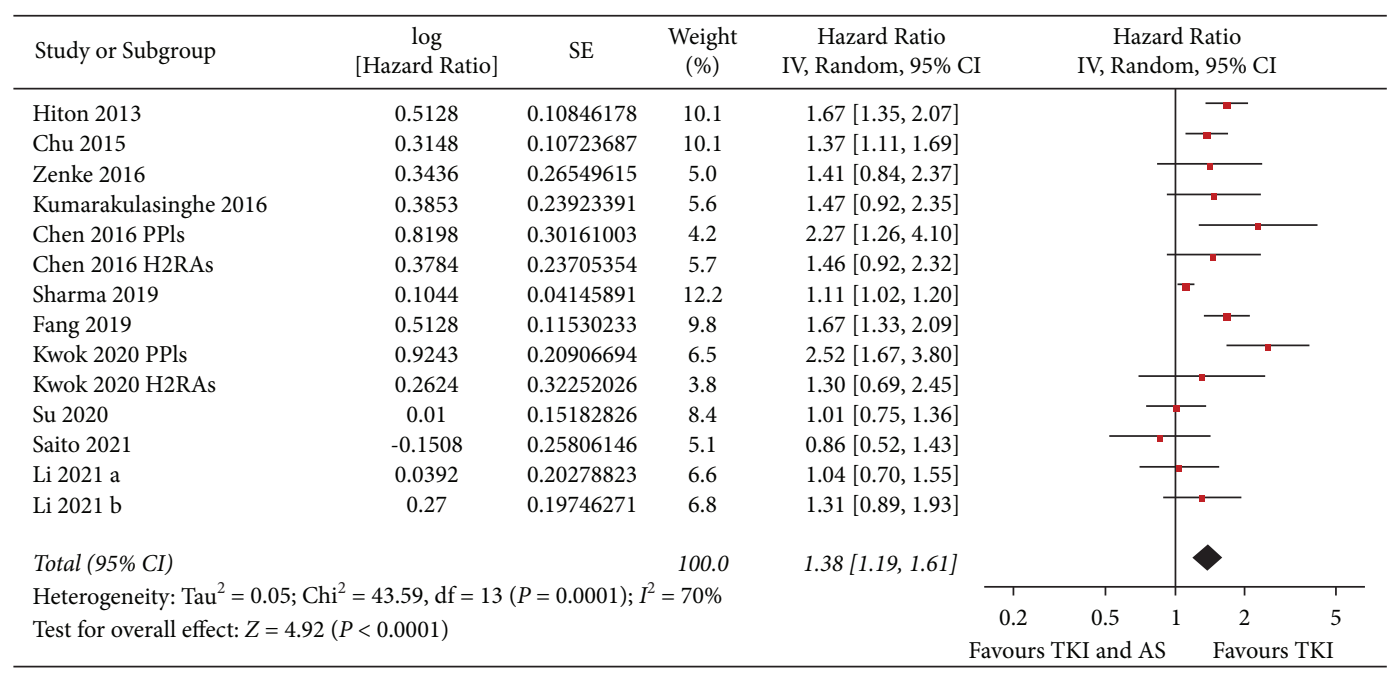

(a)

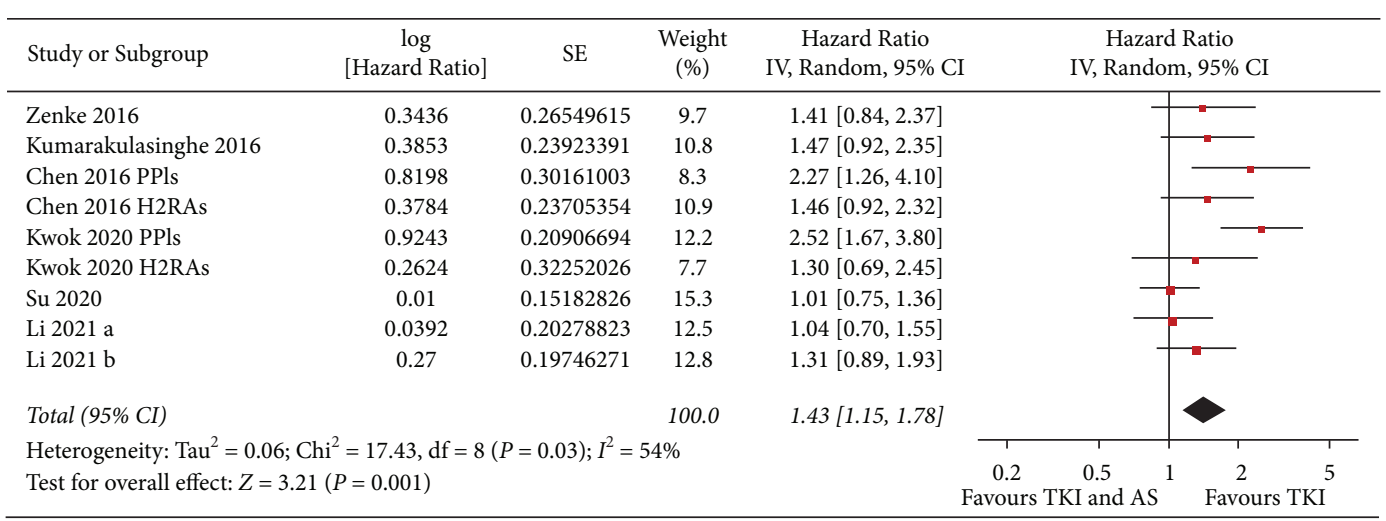

(b)

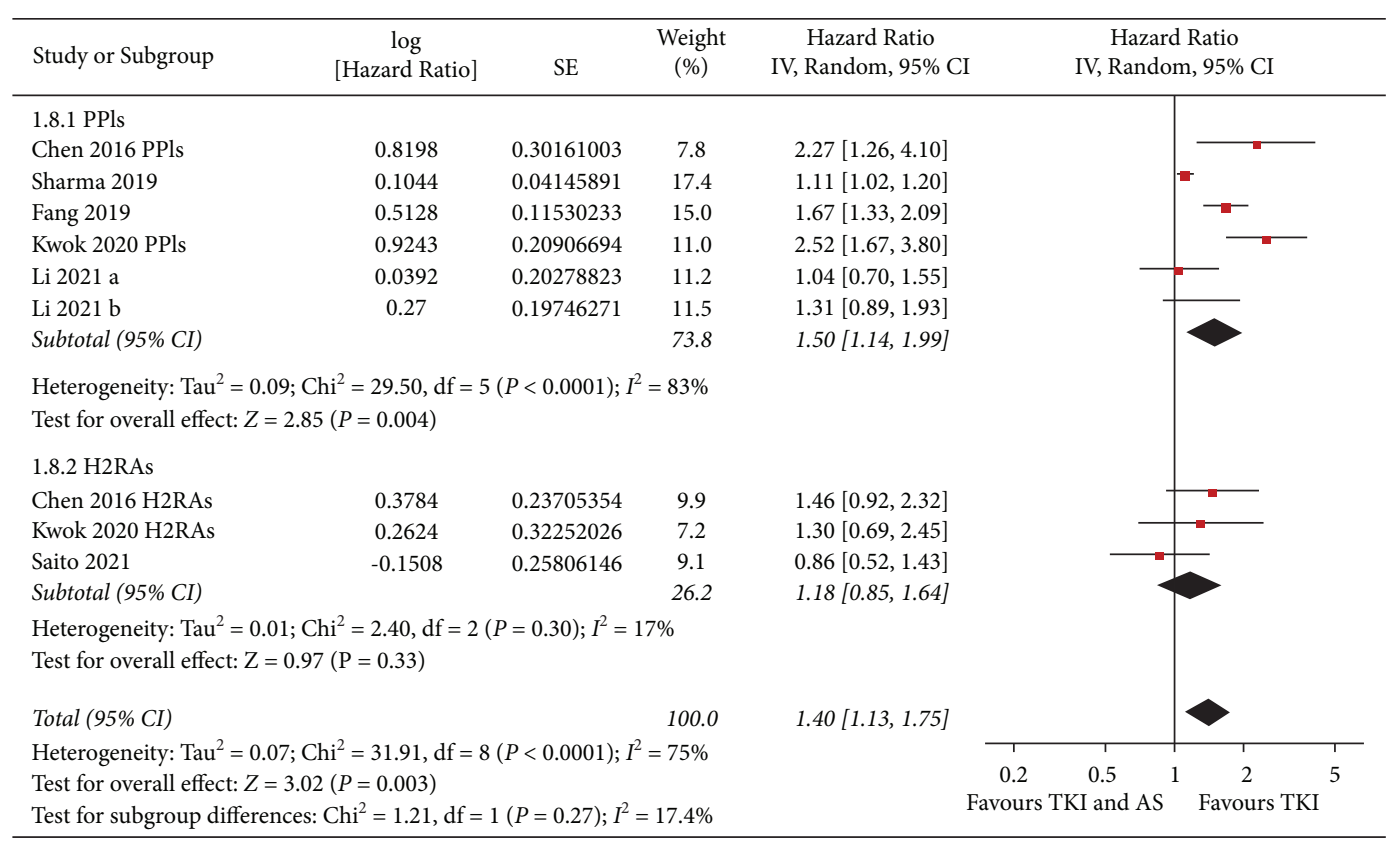

(c)

FIgURE 4: Forest plots for the meta-analysis of the association between concomitant AS use and OS in NSCLC patients taking TKIs. (a) Forest plots for the overall meta-analysis, (b) forest plots for the sensitivity analysis in patients with EGFR mutation, and (c) forest plots for the subgroup analysis according to AS used. 


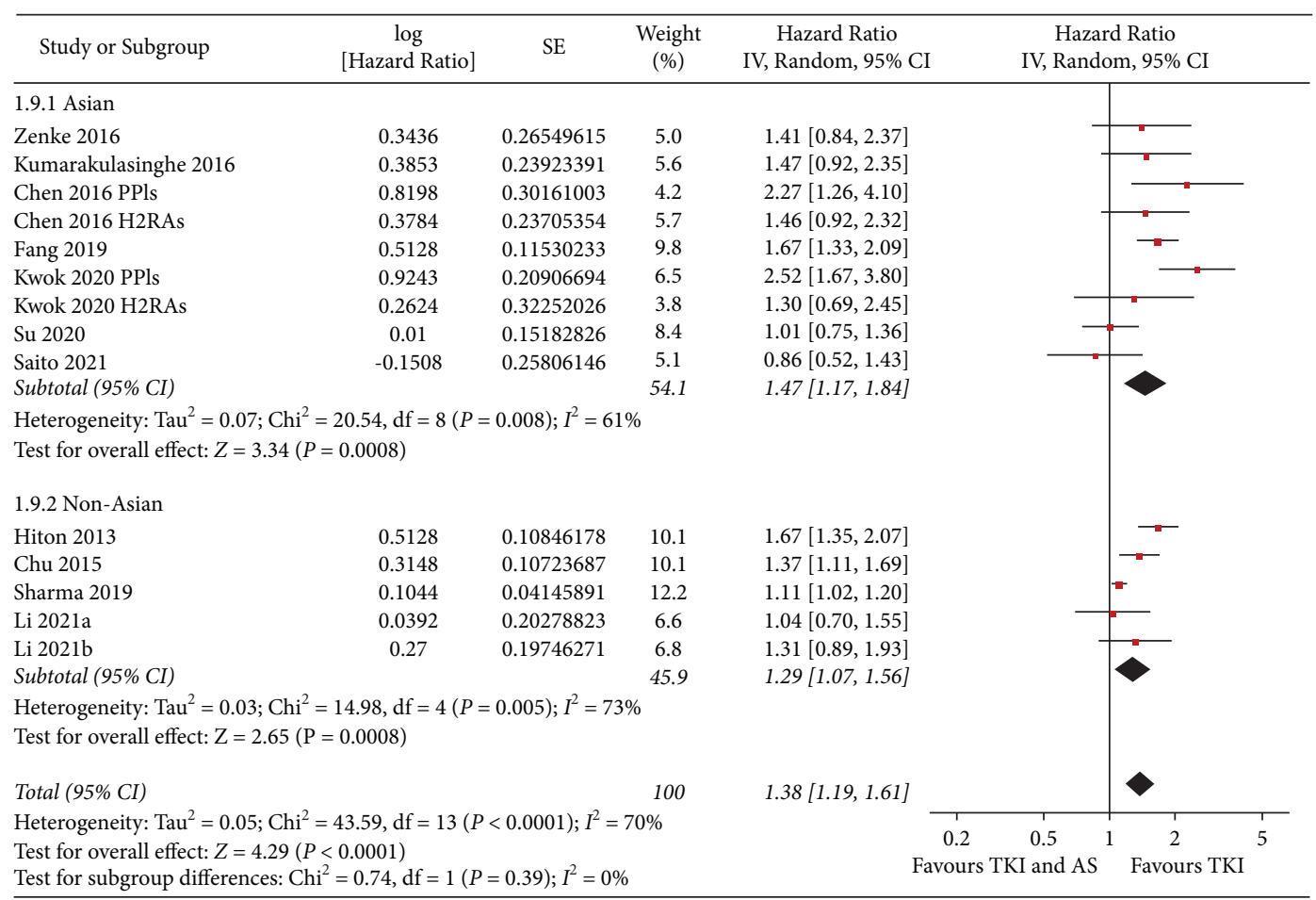

(a)

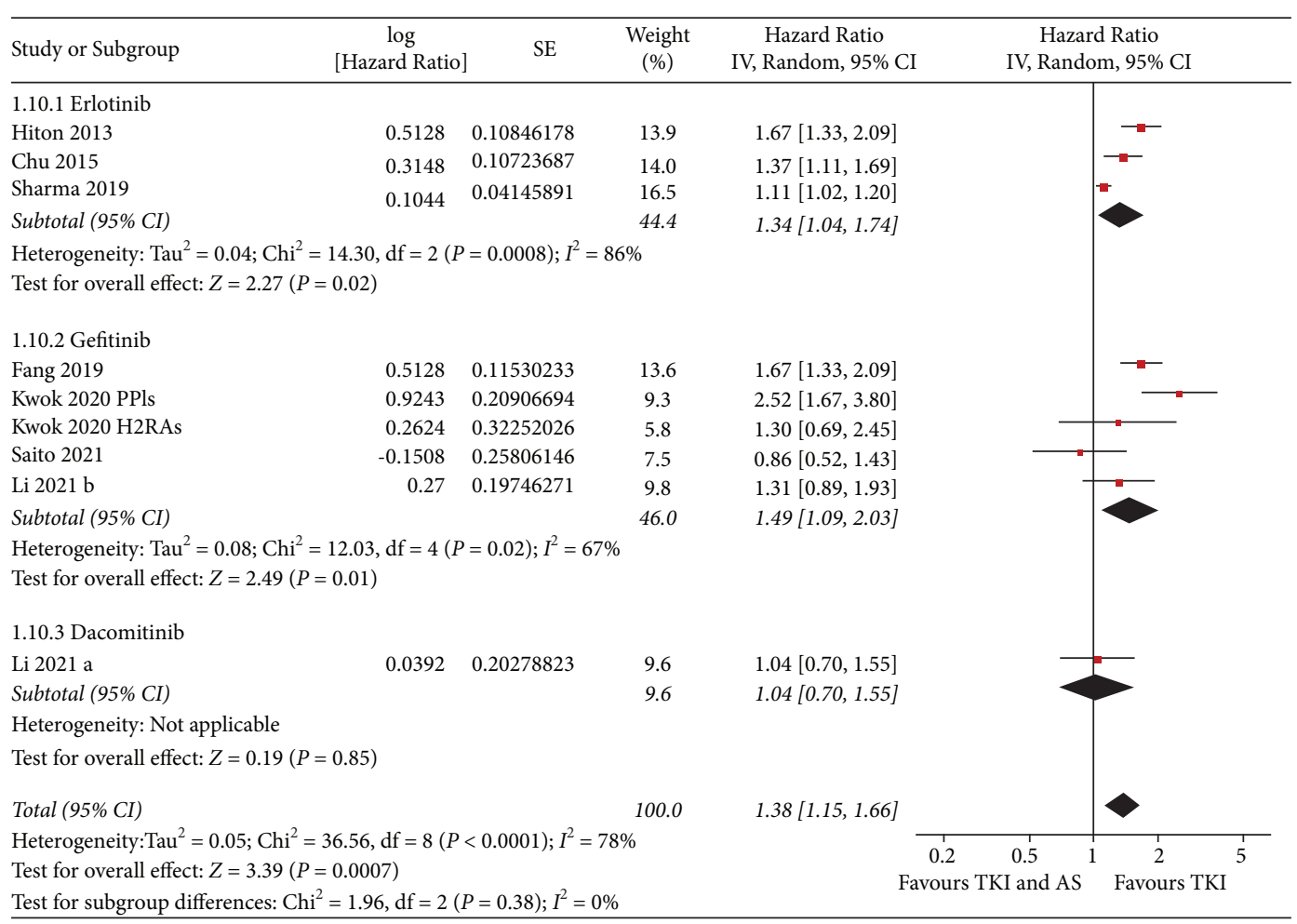

(b)

FIGURE 5: Forest plots for the subgroup analyses of the association between concomitant AS use and OS in NSCLC patients taking TKIs. (a) Subgroup analysis according to the country of the study and (b) subgroup analysis according to TKIs used. 


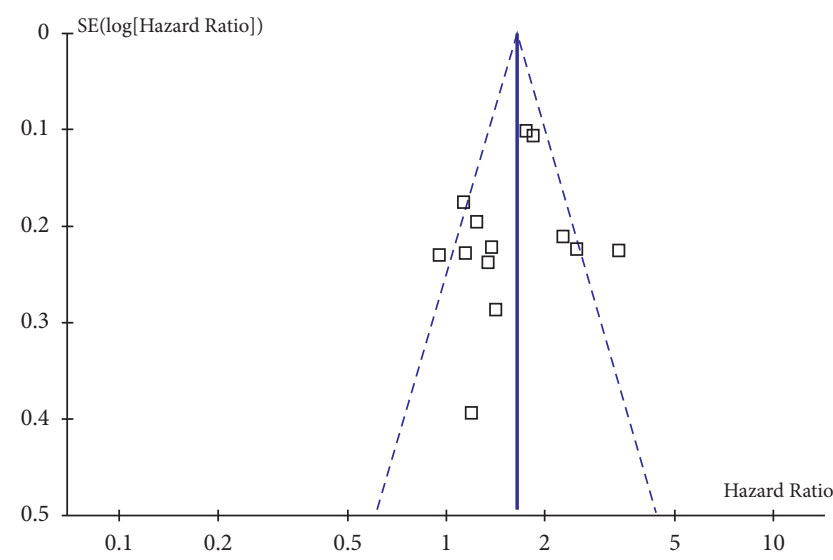

(a)

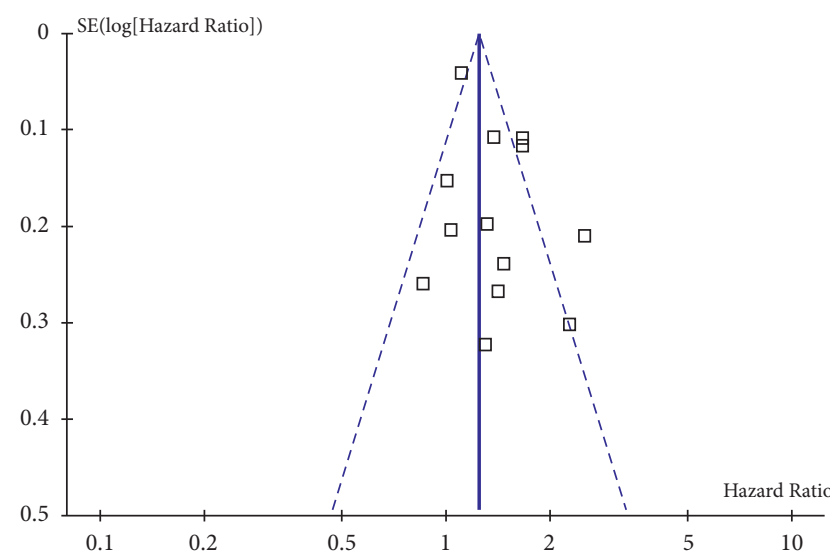

(b)

Figure 6: Funnel plots for the publication bias underlying the meta-analyses. (a) funnel plots for the meta-analysis of the association between concomitant AS use and PFS in NSCLC patients taking TKIs and (b) funnel plots for the meta-analysis of the association between concomitant AS use and OS in NSCLC patients taking TKIs.

the plasma level of osimertinib, a third-generation TKIs, was not determined by coadministration with food or PPIs [37]. Future studies are warranted to determine the influence of concomitant AS use on the survival of NSCLC patients receiving these TKIs [38].

Our study has limitations, too. Firstly, all of the included studies were retrospective, which may expose the metaanalysis to a higher risk of recall and selection biases. Largescale prospective cohort studies are needed to validate the findings. Besides, as previously mentioned, the results of the meta-analysis were primarily derived from studies with gefitinib and erlotinib. Future studies are needed to determine the influence of concomitant AS use on the survival of NSCLC patients receiving other EGFR-TKIs, such as afatinib, dacomitinib, and osimertinib. In addition, subgroup analyses should be interpreted with caution because of the limited datasets available for each subgroup. Moreover, influences of patient characteristics on the association between concomitant AS use and poor survival could not be fully analyzed in this study since it is a meta-analysis based on data from the study level. A meta-analysis based on individual patient data may be considered. In addition, for studies that were included in sensitivity analyses limited to patients with EGFR mutation [13-15, 19, 21, 22], only patients with EGFR mutations were included but not for patients without EGFR mutation. For other studies that did not specify the EGFR mutational status of the patients $[11,12,16-19,23]$, both patients with and without EGFR mutation were included. However, no subgroup data according to EGFR mutational status were provided in these studies. Accordingly, we could not perform subgroup analyses to compare the associations between patients with and without EGFR mutation. Future studies are warranted to determine whether the EGFR mutational status could affect the association between AS use and survival in NSCLC patients taking EGFR-TKIs. Finally, although we included only studies with multivariate analyses, there might be residual uncontrolled factors that may also confound the association, such as the dietary factors, other concurrent medications, and the time gap between administration of AS and TKIs.

\section{Conclusions}

In conclusion, results of this meta-analysis showed that current evidence based on retrospective studies suggested that concomitant use of AS may be independently associated with poor survival in NSCLC patients taking EGFR-TKIs such as gefitinib and erlotinib. The combined use of AS and TKIs in patients with NSCLC should be done with caution. Large-scale prospective cohort studies are needed to validate these findings and to clarify whether the type of AS and TKIs may affect the association.

\section{Data Availability}

The data used to support the findings of this study are available from the corresponding author upon request.

\section{Additional Points}

Review Criteria. (i) A comprehensive search strategy using combined keywords was designed. (ii) Medline, Embase, and Web of Science were searched up to June 10, 2021. (iii) Studies evaluating the association between concomitant AS use and survival outcomes in NSCLC patients taking TKIs were included. Message for the Clinic. (i) Concomitant use of AS may be independently associated with poor survival in NSCLC patients taking EGFR-TKIs. (ii) Combined use of AS and TKIs in patients with NSCLC should be cautious.

\section{Conflicts of Interest}

The authors declare that they have no potential conflicts of interest. 


\section{Authors' Contributions}

Jun Xia and Shiqin Xu designed the study. Jun Xia and Jiping Zhu performed a literature search, quality evaluation, and data extraction. Jun Xia, Jiping Zhu, and Lei Li performed statistical analyses and interpreted the results. Jun Xia drafted the manuscript, and Shiqin $\mathrm{Xu}$ critically revised the draft. All authors approved the submission of the manuscript.

\section{Acknowledgments}

This study was supported by the Six Talent Peaks in Jiangsu Province (No. 2015-WSW-059) and the Peak Academic Talents Plan in Jiangsu Province Hospital of Chinese Medicine (No. k2018yrc29).

\section{References}

[1] W. Shalata, B. M. Jacob, and A. Agbarya, "Adjuvant treatment with tyrosine kinase inhibitors in epidermal growth factor receptor mutated non-small-cell lung carcinoma patients, past, present and future," Cancers (Basel).vol. 13, no. 16, 2021.

[2] K. Ninomiya, S. Teraoka, Y. Zenke et al., "Japanese lung cancer society guidelines for stage IV NSCLC with EGFR mutations," JTO Clinical and Research Reports, vol. 2, no. 1, Article ID 100107, 2021.

[3] H. H. Loong, S.-C. S. Kwan, T. S.-K. Mok, and Y.-M. Lau, "Therapeutic strategies in EGFR mutant non-small cell lung cancer," Current Treatment Options in Oncology, vol. 19, no. 11, p. 58,2018

[4] Y. K. Shim and N. Kim, "The effect of H2Receptor antagonist in acid inhibition and its clinical efficacy," The Korean Journal of Gastroenterology, vol. 70, no. 1, pp. 4-12, 2017.

[5] P. Malfertheiner, A. Kandulski, and M. Venerito, "Protonpump inhibitors: understanding the complications and risks," Nature Reviews Gastroenterology \& Hepatology, vol. 14, no. 12, pp. 697-710, 2017.

[6] G. S. Smelick, T. P. Heffron, L. Chu et al., "Prevalence of acidreducing agents (ARA) in cancer populations and ARA drugdrug interaction potential for molecular targeted agents in clinical development," Molecular Pharmaceutics, vol. 10, no. 11, pp. 4055-4062, 2013.

[7] D. A. Sabbah, R. Hajjo, and K. Sweidan, "Review on epidermal growth factor receptor (EGFR) structure, signaling pathways, interactions, and recent updates of EGFR inhibitors," Current Topics in Medicinal Chemistry, vol. 20, no. 10, pp. 815-834, 2020.

[8] H. Yokota, K. Sato, Y. Okuda et al., "Effects of histamine 2receptor antagonists and proton pump inhibitors on the pharmacokinetics of gefitinib in patients with non-small-cell lung cancer," Clinical Lung Cancer, vol. 18, no. 6, pp. e433-e439, 2017.

[9] M. Ohgami, T. Kaburagi, A. Kurosawa et al., "Effects of proton pump inhibitor coadministration on the plasma concentration of erlotinib in patients with non-small cell lung cancer," Therapeutic Drug Monitoring, vol. 40, no. 6, pp. 699-704, 2018.

[10] A. A. T. Uchiyama, P. A. I. A. Silva, M. S. M. Lopes et al., "Proton pump inhibitors and oncologic treatment efficacy: a practical review of the literature for oncologists," Current Oncology, vol. 28, no. 1, pp. 783-799, 2021.
[11] J. F. Hilton, D. Tu, L. Seymour, F. A. Shepherd, and P. A. Bradbury, "An evaluation of the possible interaction of gastric acid suppressing medication and the EGFR tyrosine kinase inhibitor erlotinib," Lung Cancer, vol. 82, no. 1, pp. 136-142, 2013.

[12] M. P. Chu, S. Ghosh, C. R. Chambers et al., "Gastric Acid suppression is associated with decreased erlotinib efficacy in non-small-cell lung cancer," Clinical Lung Cancer, vol. 16, no. 1, pp. 33-39, 2015.

[13] Y.-M. Chen, C.-H. Lai, H.-C. Chang et al., "Antacid use and de novo brain metastases in patients with epidermal growth factor receptor-mutant non-small cell lung cancer who were treated using first-line first-generation epidermal growth factor receptor tyrosine kinase inhibitors," PLoS One, vol. 11, no. 2, Article ID e0149722, 2016.

[14] N. B. Kumarakulasinghe, N. Syn, Y. Y. Soon et al., "EGFR kinase inhibitors and gastric acid suppressants in EGFRmutant NSCLC: a retrospective database analysis of potential drug interaction," Oncotarget, vol. 7, no. 51, pp. 85542-85550, 2016.

[15] Y. Zenke, K. Yoh, S. Matsumoto et al., "Clinical impact of gastric acid-suppressing medication use on the efficacy of erlotinib and gefitinib in patients with advanced non-smallcell lung cancer harboring EGFR mutations," Clinical Lung Cancer, vol. 17, no. 5, pp. 412-418, 2016.

[16] M. Nieves Sedano, J. Manuel Caro Teller, C. Garcia Munoz et al., "Clinical impact of gastric acid suppressing medication on the effectiveness of tyrosine kinase inhibitors in lung cancer patients," Journal of the Balkan Union of Oncology, vol. 23, no. 3, pp. 647-653, 2018.

[17] Y.-H. Fang, Y.-H. Yang, M.-J. Hsieh, M.-S. Hung, and Y.-C. Lin, "Concurrent proton-pump inhibitors increase risk of death for lung cancer patients receiving 1st-line gefitinib treatment-a nationwide population-based study," Cancer Management and Research, vol. 11, pp. 8539-8546, 2019.

[18] M. Sharma, H. M. Holmes, H. B. Mehta et al., "The concomitant use of tyrosine kinase inhibitors and proton pump inhibitors: prevalence, predictors, and impact on survival and discontinuation of therapy in older adults with cancer," Cancer, vol. 125, no. 7, pp. 1155-1162, 2019.

[19] Z. Guo, Q. Du, X. Ye et al., "Concomitant administration of gastric acid suppression might attenuates the clinical efficacy of gefitinib: a single cancer center retrospective study," Journal of Chinese Pharmaceutical Sciences, vol. 29, no. 3, pp. 192-198, 2020.

[20] W. C. Kwok, J. C. Man Ho, D. C. Leung Lam, M. M. Sze Lui, M. S. Man Ip, and T. C. Chun Tam, "Clinical impact of gastric acid suppressants use on the efficacy of gefitinib in patients with advanced adenocarcinoma of the lung harboring common egfr mutations," Clinical Cancer Drugs, vol. 7, no. 1, pp. $57-61,2020$.

[21] V. Y.-F. Su, K.-Y. Yang, T.-Y. Huang et al., "The efficacy of first-line tyrosine kinase inhibitors combined with co-medications in Asian patients with EGFR mutation non-small cell lung cancer," Scientific Reports, vol. 10, no. 1, p. 14965, 2020.

[22] J. Li, D. Nickens, K. Wilner, and W. Tan, "Evaluation of the effect of proton pump inhibitors on the efficacy of dacomitinib and gefitinib in patients with advanced non-small cell lung cancer and EGFR-activating mutations," Oncology and Therapy, vol. 9, no. 2, 2021.

[23] Y. Saito, Y. Takekuma, M. Kobayashi et al., "Impact of histamine type-2 receptor antagonists on the anticancer efficacy of gefitinib in patients with non-small cell lung cancer," 
European Journal of Clinical Pharmacology, vol. 77, no. 3, pp. 381-388, 2021.

[24] D. F. Stroup, J. A. Berlin, S. C. Morton et al., "Meta-analysis of observational studies in epidemiologyA proposal for reporting," JAMA, vol. 283, no. 15, pp. 2008-2012, 2000.

[25] J. Higgins and S. Green, Cochrane Handbook for Systematic Reviews of Interventions Version 5.1.0, The Cochrane Collaboration, London, UK, 2011, https://www. cochranehandbook.org.

[26] G. A. Wells, B. Shea, D. O'Connell et al., "The newcastleottawa scale (NOS) for assessing the quality of nonrandomised studies in meta-analyses," 2010, https://www. ohri.ca/programs/clinical_epidemiology/oxford.asp.

[27] J. P. T. Higgins and S. G. Thompson, "Quantifying heterogeneity in a meta-analysis," Statistics in Medicine, vol. 21, no. 11, pp. 1539-1558, 2002.

[28] N. A. Patsopoulos, E. Evangelou, and J. P. Ioannidis, "Sensitivity of between-study heterogeneity in meta-analysis: proposed metrics and empirical evaluation," International Journal of Epidemiology, vol. 37, no. 5, pp. 1148-1157, 2008.

[29] A. Indini, F. Petrelli, G. Tomasello et al., "Impact of use of gastric-acid suppressants and oral anti-cancer agents on survival outcomes: a systematic review and meta-analysis," Cancers, vol. 12, no. 4, 2020.

[30] P. S. Malik, D. Jain, and L. Kumar, "Epidermal growth factor receptor tyrosine kinase inhibitors in advanced non-small cell lung cancer," Oncology, vol. 91, no. 1, pp. 26-34, 2016.

[31] Z. Yang, A. Hackshaw, Q. Feng et al., "Comparison of gefitinib, erlotinib and afatinib in non-small cell lung cancer: a meta-analysis," International Journal of Cancer, vol. 140, no. 12, pp. 2805-2819, 2017.

[32] M. Egger, G. D. Smith, M. Schneider, and C. Minder, "Bias in meta-analysis detected by a simple, graphical test," BMJ, vol. 315, no. 7109, pp. 629-634, 1997.

[33] C. B. Begg and M. Mazumdar, "Operating characteristics of a rank correlation test for publication bias," Biometrics, vol. 50, no. 4, pp. 1088-1101, 1994.

[34] N. R. Budha, A. Frymoyer, G. S. Smelick et al., "Drug absorption interactions between oral targeted anticancer agents and PPIs: is pH-dependent solubility the achilles heel of targeted therapy?" Clinical Pharmacology \& Therapeutics, vol. 92, no. 2, pp. 203-213, 2012.

[35] L. Zhang, F. Wu, S. C. Lee, H. Zhao, and L. Zhang, "pHdependent drug-drug interactions for weak base drugs: potential implications for new drug development," Clinical Pharmacology \& Therapeutics, vol. 96, no. 2, pp. 266-277, 2014.

[36] S. Peters, S. Zimmermann, and A. A. Adjei, "Oral epidermal growth factor receptor tyrosine kinase inhibitors for the treatment of non-small cell lung cancer: comparative pharmacokinetics and drug-drug interactions," Cancer Treatment Reviews, vol. 40, no. 8, pp. 917-926, 2014.

[37] K. Vishwanathan, P. A. Dickinson, K. Bui et al., "The effect of food or omeprazole on the pharmacokinetics of osimertinib in patients with non-small-cell lung cancer and in healthy volunteers," The Journal of Clinical Pharmacology, vol. 58, no. 4, pp. 474-484, 2018.

[38] R. J. Honeywell, I. Kathmann, E. Giovannetti et al., "Epithelial transfer of the tyrosine kinase inhibitors erlotinib, gefitinib, afatinib, crizotinib, sorafenib, sunitinib, and dasatinib: implications for clinical resistance," Cancers, vol. 12, no. 11, 2020 . 